ASTL.CLS 04.02.12

\title{
Cosmological parameters constraints from galaxy cluster mass function measurements in combination with other cosmological data
}

\author{
R. A. Burenin*1, A. A. Vikhlinin ${ }^{1,2}$ \\ ${ }^{1}$ Space Research Institute (IKI), Moscow \\ ${ }^{2}$ Harvard-Smithsonian Center for Astrophysics (CfA), Boston, USA
}

Received Dec. 1, 2011

\begin{abstract}
We present the cosmological parameters constraints obtained from the combination of galaxy cluster mass function measurements (Vikhlinin et al., 2009a,b) with new cosmological data obtained during last three years: updated measurements of cosmic microwave background anisotropy with Wilkinson Microwave Anisotropy Probe (WMAP) observatory, and at smaller angular scales with South Pole Telescope (SPT), new Hubble constant measurements, baryon acoustic oscillations and supernovae Type Ia observations.

New constraints on total neutrino mass and effective number of neutrino species are obtained. In models with free number of massive neutrinos the constraints on these parameters are notably less strong, and all considered cosmological data are consistent with non-zero total neutrino mass $\Sigma m_{\nu} \approx 0.4 \mathrm{eV}$ and larger than standard effective number of neutrino species, $N_{\text {eff }} \approx 4$. These constraints are compared to the results of neutrino oscillations searches at short baselines.

The updated dark energy equation of state parameters constraints are presented. We show that taking in account systematic uncertainties, current cluster mass function data provide similarly powerful constraints on dark energy equation of state, as compared to the constraints from supernovae Type Ia observations.
\end{abstract}

Key words: cosmology, cosmological parameters, galaxy clusters

\section{INTRODUCTION}

The measurements of galaxy cluster mass function give one of the most sensitive method to measure the cosmological parameters, in particular the parameters of dark energy equation of state (e.g., Starobinsky, 1998; Haiman et al., 2001; Weller et al., 2002; Wang et al., 2004; Majumdar, Mohr, 2004). Current observational data provide measurements of cluster mass function accurate enough to obtain powerful constraints on the parameters of cosmological model (e.g., Borgani et al., 2001; Henry, 2000, 2004; Reiprich, Böhringer, 2002; Vikhlinin et al., 2003; Schuecker et al., 2003; Voevodkin, Vikhlinin, 2004; Mantz et al., 2008, 2010a; Vikhlinin et al., 2009b; Vanderlinde et al., 2010; Vikhlinin, 2010).

The strongest to date constraints on cosmological parameters using the data on measurements of galaxy cluster mass function were obtained in Vikhlinin et al. (2009b). These constraints appears to be similarly powerful as compared to the constraints from most recent cosmic microwave background (CMB) measurements made by WMAP, observations of baryon acoustic oscillations and supernovae Type Ia. These new constraints are independent and have different cosmological parameter degeneracies.

\footnotetext{
e-mail: rodion@hea.iki.rssi.ru
}

In this work the confirmation of the existence of the dark energy was, for the first time, obtained using new independent method, based on the measurements of the large scale structure growth rate, not only on the measurements of the geometry of Universe. The joint analysis with other cosmological data allowed to significantly improve the measurements of the dark energy equation of state parameters and also to improve constraints on other cosmological parameters, e.g. total mass of light neutrinos.

In the last years a significant amount of new cosmological data were published. These include seven-year data of CMB observations with WMAP (Larson et al., 2011), and CMB observations at smaller angular scales (Reichardt et al., 2009; Brown et al., 2009; Dunkley et al., 2010; Keisler et al., 2011). Using the calibration of supernovae Ia absolute luminosities the measurement of Hubble constant was significantly improved Riess et al., 2009, 2011). Also the new data on observations of supernovae Ia (Hicken et al., 2009; Amanullah et al., 2010), and baryon acoustic oscillations (Percival et al., 2010) were obtained. Cosmological constraints from the joint analysis of these and others cosmological data were presented in Komatsu et al. (2011), Keisler et al. (2011) and other works.

In this paper we present the cosmological parameters 
constraints, obtained in result of joint analysis of galaxy cluster mass function measurements Vikhlinin et al., 2009a b) and recent cosmological data discussed above. As compared to Vikhlinin et al. (2009b), wider set of cosmological parameters is considered. To calculate joint likelihood functions in cosmological parameter space we used Markov Chain Monte Carlo technique (see, e.g., Lewis, Bridle, 2002). In order to include galaxy cluster mass function cosmological data in these calculations, correspondent software was developed, which we provide for public use.

In our work the new significant constraints on total neutrino mass and effective number of neutrino species are presented. In order to test the possibility of the existence of light sterile neutrinos with masses near $1 \mathrm{eV}$, which were suggested to explain the results of LSDN (Aguilar et al., 2001) and MiniBooNE (Aguilar-Arevalo A. A. et al., 2010) experiments, and also recently discovered so-called reactor neutrino anomaly (Mueller et al., 2011; Mention et al., 2011), we consider the models with free number of massive neutrinos. Also in this paper we give the updated constraints on the parameters of the dark energy equation of state. We show, that taking in account systematic uncertainties, dark energy equation of state constraints based on the existing cluster mass function data and supernovae Type Ia observations are comparably powerful.

\section{COSMOLOGICAL DATA}

In our work the data on cluster mass function measurements are taken with no changes from Vikhlinin et al. (2009b). In this work cluster mass function was measured using a sample of 86 massive galaxy clusters with masses measured using Chandra X-ray observations with about $10 \%$ accuracy (Vikhlinin et al., 2009a). Distant $(z \approx 0.4-$ $0.9)$ clusters in this sample were selected in 400 square degree X-ray galaxy cluster survey, based on ROSAT PSPC pointed data (Burenin et al., 2007). Clusters in local Universe $(z<0.2)$ were selected from ROSAT All Sky Survey (see details in Vikhlinin et al., 2009a). Confidence regions for different cosmological parameters were obtained from the analysis of likelihood function, which was calculated on a grid of cosmological parameters. The results of these calculations are available on $\mathrm{WWW}^{1}$. This cosmological data set is designated below as $C L$.

In our work we used also new cosmological data, which were published after the issue of paper by Vikhlinin et al. (2009b). The most significant improvement was achieved in the measurement of Hubble constant, due to the calibration of supernovae Ia absolute magnitudes, which was made using the Cepheid observations in SNe Ia host galaxies. After that from the observations of nearby SNe Ia the measurement $H_{0}=73.8 \pm 2.4 \mathrm{~km} \mathrm{~s}^{-1} \mathrm{Mpc}^{-1}$ was obtained (Riess et al., 2011). These cosmological data are desig-

\footnotetext{
${ }^{1}$ http://hea.iki.rssi.ru/400d/cosm/
}

nated below as $H_{0}$. This is much better measurement as compared to $H_{0}=72 \pm 8 \mathrm{~km} \mathrm{~s}^{-1} \mathrm{Mpc}^{-1}$ (Freedman et al., 2001) used by Vikhlinin et al. (2009b), and also as compared to the measurement $H_{0}=74.2 \pm 3.6 \mathrm{~km} \mathrm{~s}^{-1} \mathrm{Mpc}^{-1}$ (Riess et al., 2009) used by Komatsu et al. (2011).

In our work we used seven-year data of CMB observations from WMAP (Larson et al., 2011). For these data likelihood were calculated using the software from $L A M B D A$ archive, version $4.1^{2}$. Theoretical $\mathrm{CMB}$ anisotropy spectra were calculated using CAMB software, version January 2011, where recombination was calculated using RECFAST software (Seager et al., 1999), version 1.5, which incorporate a number of improvements in hydrogen and helium recombination theory, obtained during the last years (e.g., Dubrovich, Grachev 2005; Chluba, Sunvaev 2006, 2007, 2009, 2010; Sunyaev, Chluba 2009; Kholupenko et al. 2007, 2008). This cosmological data set is designated as WMAP7.

Also we used recently published data on CMB anisotropy at smaller angular scales obtained with South Pole Telescope (SPT, Keisler et al., 2011). The data were taken from the web page of the project $^{3}$. The contributions of "poisson" and "clustered" sources were added to the theoretical CMB anisotropy power spectra according to the directions in $\S 4.1$ of paper by Keisler et al. (2011). The templates for these components were also taken from the project web page. This data set is designated below as SPT.

In addition we used the data on baryon acoustic oscillation measurements from the data Sloan Digital Sky Survey, Data Release 7, and the data of $2 d F$ GRS survey. These data were taken as Gaussian priors for the ratio of sound horizon at the baryon-drag epoch to effective distance measure for two redshifts $z=0.2$ and $z=0.35$ (Percival et al., 2010; Komatsu et al., 2011). This data set is designated as BAO. In order to study the dark energy equation of state we also used the data on SNe Ia observations, the UNION2 ${ }^{4}$ compilation (Amanullah et al., 2010). This data set is designated below as $S N$.

\section{LIKELIHOOD CALCULATION}

In Vikhlinin et al. (2009b) likelihood functions for galaxy cluster mass function data were calculated at cosmological parameters grids, since in that case these calculations could be performed very effectively. In order to use these likelihood calculations in Markov Chains Monte Carlo simulations in multidimensional cosmological parameters space (Lewis, Bridle, 2002), we wrote a module for CosmoMC ${ }^{5}$ software, where likelihood in arbitrary

\footnotetext{
${ }^{2}$ http://lambda.gsfc.nasa.gov/product/map/dr4/likelihood_get.cfm

${ }^{3}$ http://pole.uchicago.edu/public/data/keisler11/

${ }^{4} \mathrm{http}: / /$ supernova.lbl.gov/Union/

${ }^{5}$ http://cosmologist.info/cosmomc/
} 
point of parameters space is calculated using simple interpolation on parameters grids. In spite of ideological simplicity, the realization turn out be somewhat cumbersome due to many dimension grids. Therefore, we think it may be useful to provide this module for public use. This software can be found at web page of $C C C P$ project $^{6}$.

Using this module one can easily include likelihood for galaxy cluster mass function from Vikhlinin et al. (2009b) in calculation of Markov Chains with CosmoMC software. Also one can use this module to include this likelihood into existing parameters chains using priors adjustment procedure. In our work below we mainly used our own calculations of Markov Chains with CosmoMC software. In some cases we also used chains, version WMAP7.2, taken from LAMBDA archive ${ }^{7}$.

The constraints for some cosmological parameters in various cosmological models are presented in Table 1. More extended information on cosmological constraints obtained in frames of our work can be found at correspondent web page ${ }^{8}$.

In all Figures below we give contours for $68 \%$ and $95 \%$ confidence regions. In most Figures the pair of contours of larger size corresponds to the set of cosmological data without the data on cluster mass function, the pair of contours of smaller size - with cluster mass function data taken in account. All confidence intervals are given at $68 \%$ confidence level, all upper limits — at $95 \%$ confidence level.

\section{SYSTEMATIC UNCERTAINTIES}

Systematic uncertainties of galaxy cluster mass function measurements were studied in detail by Vikhlinin et al. (2009b). They were not included in likelihood functions, calculated in this work. Thus, systematical errors for cosmological parameters constraints should be estimated separately. These uncertainties can be estimated by measuring the shifts of likelihood maximum due to variation of observables by their systematical errors.

For galaxy cluster mass function data the main source of systematical errors is the uncertainty in cluster mass measurements. The mass measurements used in our work are based on hot intracluster gas temperatures and masses and were calibrated using hydrostatic measurements of their total gravitational masses in nearby clusters (Vikhlinin et al., 2009a). Systematical error of hydrostatic mass measurements was estimated as $\delta M / M \approx 0.09$ from the comparison with the cluster mass measurements using weak lensing data taken from Hoekstra (2007) and Zhang et al. (2008).

This systematical uncertainty of cluster mass measurements gives the uncertainty $\delta \sigma_{8} \approx 0.02$ for fixed $\Omega_{m}$ (Vikhlinin et al., 2009a). When the other cosmological data are taken in account, systematical underestimation of

\footnotetext{
${ }^{6} \mathrm{http}: / /$ hea.iki.rssi.ru/400d/cosm/

${ }^{7} \mathrm{http} / / /$ lambda.gsfc.nasa.gov/product/map/dr4/parameters.cfm

${ }^{8}$ http://hea.iki.rssi.ru/400d/cosm/combined/
}
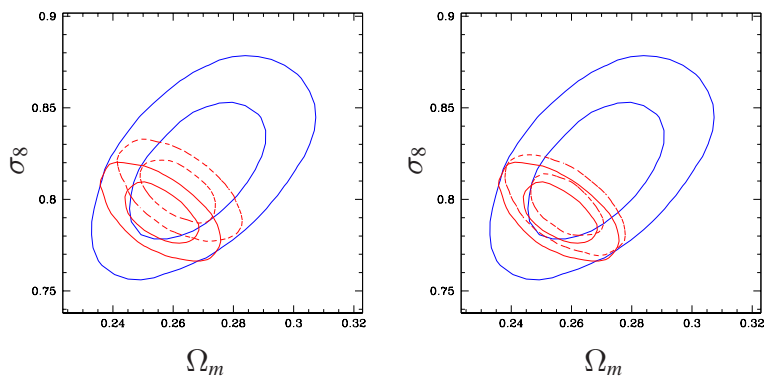

Fig. 1. Constraints for $\Omega_{m}$ and $\sigma_{8}$ in $\Lambda C D M$ model. Contours of larger size - from $W M A P 7+B A O+H_{0}$ dataset, of smaller size from $C L+W M A P 7+B A O+H_{0}$ dataset. Dashed lines show contours for $\delta M / M \approx 0.09$ systematical shift in cluster mass measurements (left), and also for the departure from self-similar evolution, $\delta M / M \approx 0.05$ for distant clusters (right).

cluster masses for $\delta M / M \approx 0.09$ leads to the shift of confidence contours, as it is shown in the left panel of Fig. 1. In this Figure, as an example, we show the constraints in $\Omega_{m}$ $-\sigma_{8}$ plane, in the model of flat Universe with cosmological constant $(\Lambda C D M)$, obtained using $C L+W M A P 7+B A O+H_{0}$ cosmological data set. In the left part of this Figure the confidence contours for $\delta M / M \approx 0.09$ systematical shift in cluster mass measurements are shown with dashed lines. Since the likelihood maximum is shifted not along the line of constant $\Omega_{m}$, the systematic error for $\sigma_{8}$ parameter turns out to be substantially smaller, $\delta \sigma_{8} \approx 0.011$ in this case. Therefore, systematic errors for cosmological parameters may be significantly reduced when the additional cosmological data are taken in account.

In addition to the error of the mass measurement calibration for nearby clusters, a significant part of total systematical error comes from the uncertainties in their cosmological evolution, which lead to the difference in mass scale calibrations for nearby and distant clusters. The systematic uncertainty due to the departure from self-similar evolution can be estimated as $\delta M / M \approx 0.05$ for distant clusters at $z \approx 0.6$ (see details in Vikhlinin et al., 2009a). This difference in mass scales corresponds to the shift of confidence contours in $\Omega_{m}-\sigma_{8}$ plane shown in right panel of Fig. 1. Quadratic sum of to systematic errors estimated for these two main uncertainties in cluster mass measurements is given below as an estimate of total systematical error for cosmological parameters constraints.

In correspondent parts of this paper the systematic uncertainties of the data on observations of supernovae Type Ia, UNION2 compilation (Amanullah et al., 2010), are also considered. Fore these data the total, systematical and statistical errors were calculated using correspondent covariance matrix. Separate estimate of systematic error for these data was obtained by quadratic subtraction of statistical error from total one. 
Table 1. Cosmological parameters constraints

\begin{tabular}{|c|c|c|c|c|}
\hline Model & Data & Parameter & Value* $^{*}$ & Systematical error \\
\hline$\Lambda C D M$ & $C L+W M A P 7+B A O+H_{0}$ & $\Omega_{m} h^{2}$ & $0.1311 \pm 0.0016$ & \pm 0.0018 \\
\hline$\ldots$ & a & $z_{e q}$ & $3155 \pm 38$ & \pm 44 \\
\hline$\ldots$ & $\ldots$ & $\sigma_{8}$ & $0.792 \pm 0.010$ & \pm 0.013 \\
\hline$\ldots$ & $\ldots$ & $\Omega_{m}$ & $0.2558 \pm 0.0077$ & \pm 0.0063 \\
\hline$\ldots$ & $\ldots$ & $H_{0}$ & $71.6 \pm 1.0 \mathrm{~km} \mathrm{~s}^{-1} \mathrm{Mpc}^{-1}$ & $\pm 0.4 \mathrm{~km} \mathrm{~s}^{-1} \mathrm{Mpc}^{-1}$ \\
\hline$\ldots$ & $\ldots$ & $\Omega_{b}$ & $0.04386 \pm 0.00086$ & \pm 0.00064 \\
\hline$\Lambda C D M$ & $C L+W M A P 7+B A O+H_{0}+S P T$ & $\Omega_{m} h^{2}$ & $0.1310 \pm 0.0014$ & \pm 0.0016 \\
\hline$\ldots$ & - & $z_{e q}$ & $3154 \pm 33$ & \pm 39 \\
\hline$\ldots$ & $\ldots$ & $\sigma_{8}$ & $0.7921 \pm 0.0089$ & \pm 0.0115 \\
\hline$\ldots$ & $\ldots$ & $\Omega_{m}$ & $0.2560 \pm 0.0067$ & \pm 0.0072 \\
\hline$\cdots$ & $\cdots$ & $H_{0}$ & $71.55 \pm 0.77 \mathrm{~km} \mathrm{~s}^{-1} \mathrm{Mpc}^{-1}$ & $\pm 0.56 \mathrm{~km} \mathrm{~s}^{-1} \mathrm{Mpc}^{-1}$ \\
\hline$\ldots$ & $\ldots$ & $\Omega_{b}$ & $0.04349 \pm 0.00075$ & \pm 0.00071 \\
\hline$\Lambda C D M+\Omega_{k}$ & $C L+W M A P 7+B A O+H_{0}+S P T$ & $\Omega_{k}$ & $-0.0018 \pm 0.0042$ & \\
\hline$\ldots$ & $\ldots$ & $\Omega_{\Lambda}$ & $0.7430 \pm 0.0072$ & \\
\hline$\Lambda C D M+m_{\nu}$ & $C L+W M A P 7+H_{0}+B A O+S P T$ & $\Sigma m_{\nu}$ & $<0.32 \mathrm{eV}$ & \\
\hline$\ldots$ & $C L+W M A P 7+H_{0}+B A O+S P T+S N$ & $\Sigma m_{\nu}$ & $<0.28 \mathrm{eV}$ & \\
\hline$\Lambda C D M+N_{\text {eff }}$ & $C L+W M A P 7+B A O+H_{0}+S P T$ & $N_{\text {eff }}$ & $<3.74$ & \\
\hline .. & $C L+W M A P 7+B A O+H_{0}+S P T+S N$ & $N_{\text {eff }}$ & $<3.70$ & \\
\hline$\Lambda C D M+m_{\nu}+N_{e f f}$ & $C L+W M A P 7+B A O+H_{0}+S P T$ & $\Sigma m_{\nu}$ & $<0.72 \mathrm{eV}$ & \\
\hline$\ldots$ & .. & $\Sigma m_{\nu}$ & $0.36 \pm 0.16^{* *} \mathrm{eV}$ & $\pm 0.08 \mathrm{eV}$ \\
\hline$\ldots$ & $\ldots$ & $N_{\text {eff }}$ & $<4.62$ & \\
\hline$W C D M$ & $C L+W M A P 7+B A O+H_{0}$ & $w$ & $-1.027 \pm 0.069$ & \pm 0.028 \\
\hline$\ldots$ & $C L+W M A P 7+B A O+H_{0}+S N$ & $w$ & $-0.990 \pm 0.034$ & $\pm 0.041^{* * *}$ \\
\hline$\ldots$ & $C L+W M A P 7+B A O+H_{0}+S P T$ & $w$ & $-1.013 \pm 0.066$ & \pm 0.029 \\
\hline$\ldots$ & $C L+W M A P 7+B A O+H_{0}+S P T+S N$ & $w$ & $-0.982 \pm 0.032$ & $\pm 0.038^{* * *}$ \\
\hline$W C D M+\Omega_{k}$ & $C L+W M A P 7+B A O+H_{0}$ & $w$ & $-1.12 \pm 0.13$ & \\
\hline$\ldots$ & $C L+W M A P 7+B A O+H_{0}+S N$ & $w$ & $-0.991 \pm 0.039$ & \\
\hline WACDM & $C L+W M A P 7+B A O+H_{0}+S N$ & $w_{0}$ & $-1.13 \pm 0.11$ & \\
\hline$\ldots$ & $\ldots$ & $w_{a}$ & $0.47 \pm 0.36$ & \\
\hline
\end{tabular}

* - all intervals are given at $68 \%$ confidence level, all upper limits — at 95\% confidence level;

** — interval is non-gaussian, for significance of non-zero value see text;

*** — including systematic uncertainty of SNe Ia data;
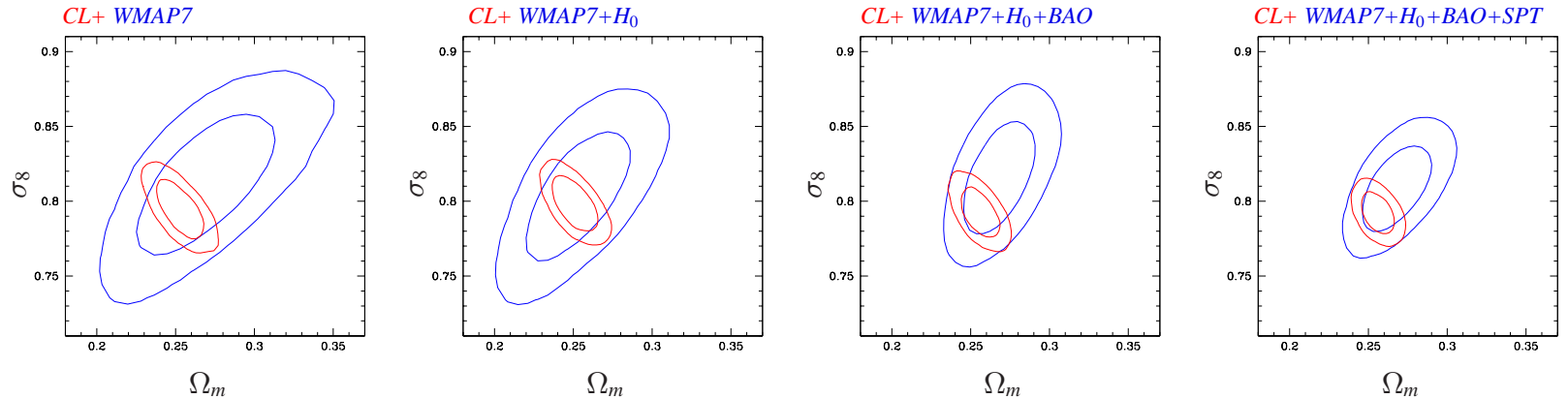

Fig. 2. Constraints on $\Omega_{m}$ and $\sigma_{8}$ in $\Lambda C D M$ model, obtained using different datasets. Larger contours, from left to the right: $W M A P 7$, $W M A P 7+H_{0}$, $W M A P 7+B A O+H_{0}, W M A P 7+B A O+H_{0}+S P T$. Smaller contours - using the same data, with the data on galaxy cluster mass function added. 

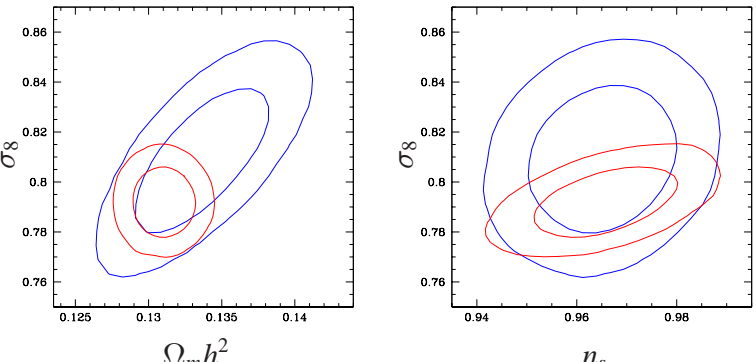

$n_{S}$
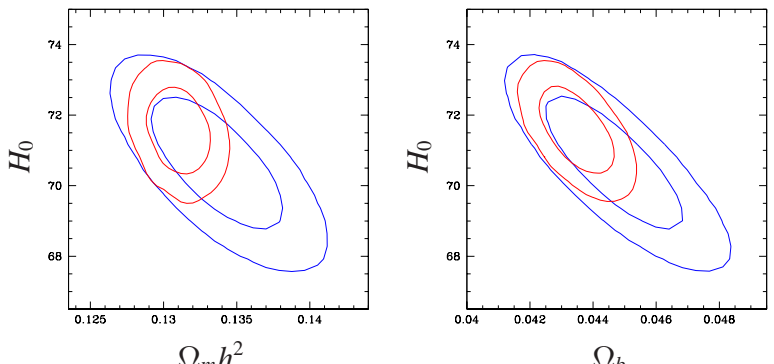

Fig. 3. Constraints on $\Omega_{m} h^{2}, \sigma_{8}, H_{0}, \Omega_{b}$ and $n_{s}$ in $\Lambda C D M$ model. Contours of larger size - from $W M A P 7+B A O+H_{0}+S P T$ dataset, of smaller size - from $C L+W M A P 7+B A O+H_{0}+S P T$ dataset.

\section{CONSTRAINTS FOR $\Lambda C D M$ MODEL}

In the upper part of Table 1 the cosmological parameters constraints for flat Universe with cosmological constant ( $\Lambda C D M$ model) are presented. The nature of main constraints is as follows. The constraints from galaxy cluster mass function are based mainly on its normalization and have their main effect on $\Omega_{m}$ and $\sigma_{8}$ - the constraints turns out to be degenerate, these two parameters are related as $\sigma_{8}=0.813\left(\Omega_{M} / 0.25\right)^{-0.47} \pm 0.011$ Vikhlinin et al. $2009 \mathrm{~b})$. The relation between $\sigma_{8}$ and CMB anisotropy amplitude depends mostly on linear perturbation growth between decoupling epoch and $z=0$ (see, e.g., approximate formula from $\mathrm{Hu}$, Jain, 2004), which in turn strongly depends on $\Omega_{m}$. Therefore, the data on CMB anisotropy amplitude allow to break the above $\Omega_{m}-\sigma_{8}$ degeneracy and these data analyzed jointly provide most accurate measurement of both $\sigma_{8}$ and $\Omega_{m}$.

The relative power of various datasets to constrain $\Omega_{m}$ and $\sigma_{8}$ parameters is shown in Fig. 2 One can see that the main power of constraints in $\triangle C D M$ model indeed originate from the joint analysis of galaxy cluster mass function and CMB data. The other cosmological data give only smaller improvement to the joint constraints on $\sigma_{8}$ and $\Omega_{m}$.

Parameters $\sigma_{8}$ and $\Omega_{m} h^{2}$ appear to be best constrained from joint analysis of all cosmological data considered in our work, which is shown in Fig. 3 The projection of these constraints on other parameters significantly improve their measurements in some cases. For example, the constraints are improved for parameters $H_{0}$ and $\Omega_{b}$. However, the data on cluster mass function do not improve significantly, for example, the constraints on the combination $\Omega_{b} h^{2}$, or on

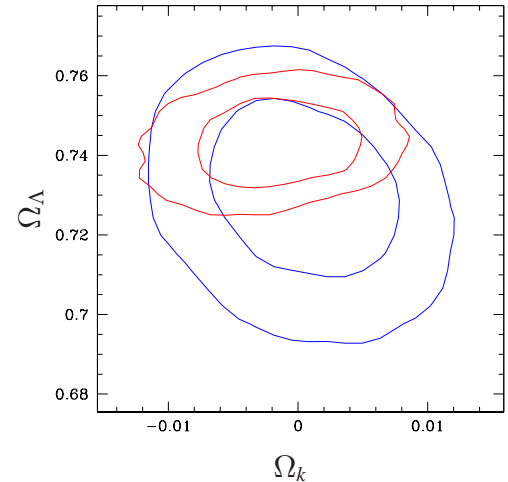

Fig. 4. Constraint on spacial curvature in Universe, $\Omega_{k}$, in $\Lambda C D M$ model with $\Omega_{k} \neq 0$. Contours are the same as in Fig. 3

spectral index of density perturbations $n_{s}$.

Note, that in considered cosmological model it is suggested that there are no other relativistic particles at equipartition in addition to photons and three known neutrino species and the relativistic energy density is known exactly. Therefore, in this model the constraint on $\Omega_{m} h^{2}$ is equivalent to the constrain on equipartition redshift $z_{e q}$ (in the left panel of Fig. 3 the axis $\Omega_{m} h^{2}$ may be changed to $z_{e q}$, see also Table 1). If the number of relativistic particles is considered as free parameter, relativistic energy density is no longer exactly defined quantity and it may be measured from cosmological data. In this case, the data on galaxy cluster mass function allow to obtain significant constraints on the number of relativistic species (see below).

\section{EXTENSIONS OF $\Lambda C D M$ MODEL Spacial curvature}

Constraints on spacial curvature in $\Lambda C D M$ model with $\Omega_{k} \neq 0$, and also on the cosmological constant density are shown in Fig. 4 (see also Table 1). The constraint on $\Omega_{k}$ is not improved significantly, there is only approximately $20 \%$ improvement, as compared to the case where cluster data are not taken in account (see Fig. (4). We note, that in this model galaxy cluster data allow to better constrain $\Omega_{m}$ and $\Omega_{\Lambda}$ separately.

\section{Primordial helium abundance}

If the data on galaxy cluster mass function are added to $W M A P 7+B A O+H_{0}$ dataset, the upper limit on primordial helium abundance, $Y_{p}$, is significantly improved (see Fig. 5] left panel). However, the data on cluster mass function do not change significantly the constraints, which are obtained with $S P T$ data taken in account (Fig. 5, right panel). From $C L+W M A P 7+B A O+H_{0}+S P T$ dataset we obtain $Y_{p}=0.279 \pm 0.028$, i.e., the measurement of primordial helium abundance is shifted slightly to its standard value $Y_{p} \approx 0.25$, which is obtained from the theoretical calculations of primordial nucleosynthesis and from the measure- 

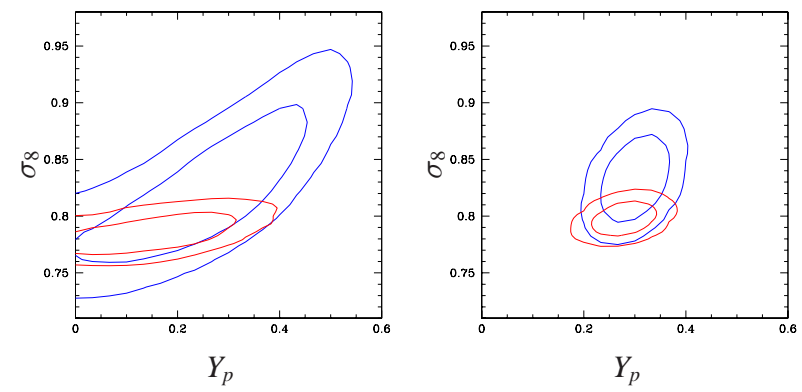

Fig. 5. Constraints on primordial helium abundance in $\triangle C D M$ model with free $Y_{p}$. Larger contours - WMAP7+BAO+H dataset (left), and from $W M A P 7+B A O+H_{0}+S P T$ dataset (right), smaller contours - using the same data, with the data on galaxy cluster mass function added.

ments of $\Omega_{b} h^{2}$ with standard value of effective number of neutrino species $N_{\text {eff }}$.

\section{Tensor modes and running spectral index}

It turns out, that existing cosmological data on galaxy cluster mass function does not improve the constraints on tensor-to-scalar ratio $r$ and running spectral index of primordial perturbations $d n_{s} / d \ln (k)$ (see Fig. 6).

\section{CONSTRAINTS ON TOTAL NEUTRINO MASS AND EFFECTIVE NUMBER OF NEUTRINO SPECIES}

\section{Massive neutrinos}

Massive neutrinos with masses of order of few $0.1 \mathrm{eV}$ would produce significant suppression of the matter density fluctuation at galaxy cluster scale since they remain to be relativistic after equipartition and are started to be involved in gravitational instability growth only later (see, e.g., Doroshkevich et al., 1981; Hu et al., 1998). This change the relation between linear perturbations amplitude $\sigma_{8}$ and normalization of CMB anisotropy power spectrum, which strongly depend also on $\Omega_{m}$. If the measurement of Hubble constant is added to the measurements of $\sigma_{8}$ and normalization of CMB anisotropy power spectrum, the $\Omega_{m}$ and total neutrino mass are both constrained with these data.

Since massive neutrinos are involved in gravitational instability considerably later than dark matter particles and baryons, their presence could change the process of nonlinear gravitational collapse of galaxy cluster haloes. The detailed analysis of this question using numerical simulations show, that these changes are indeed notable, but the correspondent change of cluster mass function appears to be not larger than few percents for neutrinos with total mass about $0.3 \mathrm{eV}$ and clusters with masses of order $10^{14} M_{\odot}$ (Brandbyge et al., 2010; Marulli et al., 2011). This is smaller that the accuracy of theoretical mass function calculations used in our work, which is approximately equal to $5 \%$ (Tinker et al., 2008).

The constraints on total neutrino mass from $C L+W M A P 7+H_{0}$ dataset are shown in the left panel
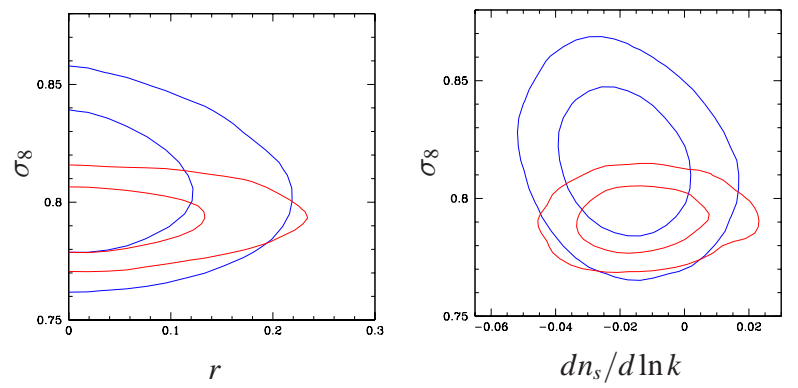

Fig. 6. Constraints on tensor-to-scalar ratio and running spectral index in correspondent extension of $\Lambda C D M$ model. Larger contours - from $W M A P 7+B A O+H_{0}+S P T$ dataset, smaller contours - from $C L+W M A P 7+B A O+H_{0}+S P T$ dataset.

of Fig. 7] the upper limit is $\Sigma m_{\nu}<0.29 \mathrm{eV}$ (95\% c. 1., see also Table 11. In the right panel of Fig. 7 we show the constrains, obtained with additional available data: $C L+W M A P 7+B A O+H_{0}+S P T$, in this case the upper limit is $\Sigma m_{\nu}<0.32 \mathrm{eV}$. If the data on SN Ia are added, the constraint is $\Sigma m_{\nu}<0.28 \mathrm{eV}$. Therefore, the additional cosmological data provide almost no changes in total neutrino mass constraints, as compared to $C L+W M A P 7+H_{0}$ dataset.

From these results we see, that new cosmological data does not significantly improve the constraints on total neutrino mass, which were obtained from the same galaxy cluster data in Vikhlinin et al. (2009b) $-\Sigma m_{\nu}<0.33 \mathrm{eV}$. Among the other recent constraints on total neutrino mass, we note the upper limit $\Sigma m_{\nu}<0.58 \mathrm{eV}$, obtained using the data on the CMB anisotropy spectrum and Hubble constant measurements (Komatsu et al., 2011). Also significant constraints were obtained from the other data on cluster mass function or density perturbations power spectrum (Malinovsky et al., 2008; Mantz et al., 2010b; Reid et al., 2010; Thomas et al., 2010). The strongest upper limits on total neutrino mass was published in papers, based on the observations of Lyman- $\alpha$ forest Seljak et al., 2006). However, the more accurate treatment of systematic errors should weaken these constraints considerably (Bolton et al., 2008). A recent review of total neutrino mass measurements from astrophysical data can be found in Abazaiian et al. (2011).

We note, that all the constraints on total neutrino mass described above were obtained in assumption that only photons and three known neutrino species are relativistic at matter-radiation equipartition epoch. It turns out that if additional neutrino species are allowed in the model, the constraints on total neutrino mass change significantly (see below).

\section{Effective number of neutrino species}

The present CMB radiation density is directly related to the density of relativistic matter at matter-radiation equipartition epoch, only if the number of relativistic parti- 

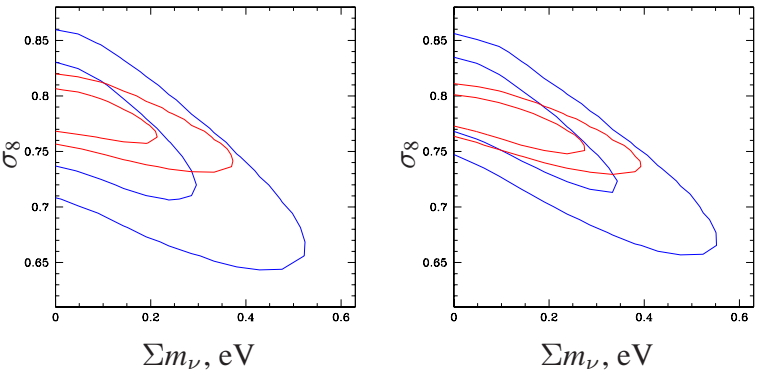

Fig. 7. Constraints on total neutrino mass in $\Lambda C D M$ model with non-zero neutrino mass. Larger contours - from $W M A P 7+H_{0}$ dataset (left) and from $W M A P 7+B A O+H_{0}+S P T$ dataset (right), smaller contours — from the same data, with the data on galaxy cluster mass function added.

cles species at equipartition is assumed to be known - a set of relativistic particles consists of photons and three known neutrino species. If the number of relativistic particles at equipartition differ from its standard value, than the relativistic matter density is not defined anymore. In this case the determination of relativistic matter density at equipartition from cosmological data gives the measurement of the effective number of relativistic species.

The number of relativistic species is usually parametrized with the number of species of light neutrinos $N_{\text {eff }}$, which gives the following relation between the densities of relativistic mater and CMB photon energy:

$$
\rho_{r}=\left[1+\frac{7}{8}\left(\frac{4}{11}\right)^{4 / 3} N_{e f f}\right] \rho_{\gamma}
$$

Note, that for the case of three known neutrino species accurate calculations of neutrino decoupling give somewhat larger effective number of neutrino species, $N_{\text {eff }}=$ 3.046 (e.g., Gnedin \& Gnedin, 1998; Dolgov et al., 1999; Mangano et al., 2002).

One of the main observables, which is measured from the CMB observations is the size of particle horizon at radiation-matter energy density equipartition and, therefore, equipartition redshift $z_{e q}$ (e.g., Hu, Dodelson, 2002; Gorbunov \& Rubakov, 2010). On the other hand, as it was shown above, galaxy cluster mass function data allow to significantly improve the measurement of $\Omega_{m} h^{2}$, using the measurement of the matter density perturbation amplitude $\sigma_{8}$ and CMB anisotropy normalization. The measurement of two parameters, $z_{e q}$ and $\Omega_{m} h^{2}$, gives the measurement of relativistic energy density at equipartition, which allow to obtain constraints on $N_{\text {eff }}$.

The constraints on the effective number of light neutrino species obtained from joint analysis of data on galaxy cluster mass function and $W M A P 7+B A O+H_{0}$ dataset is shown in left panel of Fig. 8, the upper limit is $N_{\text {eff }}<4.07$. If the data on $\mathrm{CMB}$ power spectrum at higher multipoles are added, the constraint is improved, because the damping of CMB power at small angular scales depends on $N_{\text {eff }}$ as well. This is shown in the right panel of Fig. 8, where
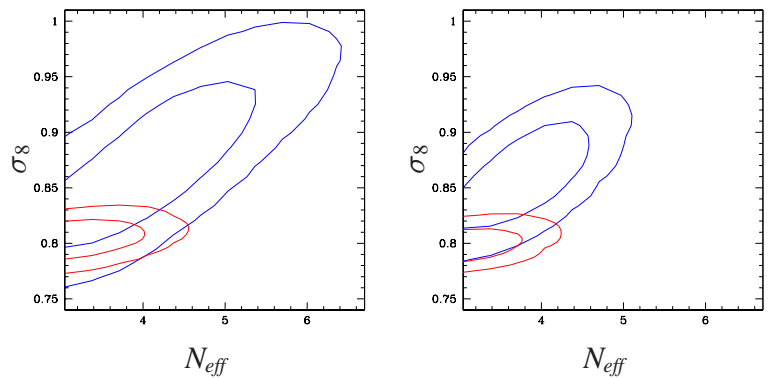

Fig. 8. Constraints on the effective number of neutrino species in $\triangle C D M$ model with free $N_{\text {eff }}$. Larger contours - from $W M A P 7+B A O+H_{0}$ dataset (left) and from emphWMAP7 $+\mathrm{BAO}+H_{0}+\mathrm{SPT}$ (right), smaller contours from the same data, with the data on galaxy cluster mass function added.

$S P T$ data are added to $W M A P 7+B A O+H_{0}$ dataset. With these data taken in account, the upper limit is $N_{\text {eff }}<3.74$. Note, that the data on galaxy cluster mass function not only improve the constraints on $N_{\text {eff }}$, but it is also shift them closer to the standard value $N_{\text {eff }}=3.046$. The data on $\mathrm{SN}$ Ia also improve this constraints slightly, in this case we obtain $N_{\text {eff }}<3.70$. Systematical errors in cluster mass measurements make these upper limits slightly less strong. For example, if cluster masses are underestimated for $\delta M / M=$ 0.09 , from $C L+W M A P 7+B A O+H_{0}+S P T$ dataset we obtain $N_{\text {eff }}<3.89$.

These results can be compared to the following constraints obtained recently. Using the results of Atacama Cosmology Telescope (ACT) observations in combination with the data on $H_{0}$ and BAO measurements, the following constraint was obtained: $N_{\text {eff }}=4.56 \pm 0.75$ (68\% c.l., Dunkley et al., 2010). Using new data of South Pole Telescope (SPT) survey together with the same measurements of $H_{0}$ and BAO, the constraint $N_{\text {eff }}=3.86 \pm 0.42$ was obtained (Keisler et al., 2011). In these constraints somewhat higher values of $N_{\text {eff }}$ are preferred, but standard value $N_{\text {eff }}=3.046$ is not ruled out at $95 \%$ confidence level.

In Keisler et al. (2011), SPT data were analyzed jointly with the data on galaxy cluster mass function in the form of constraint on $\sigma_{8}\left(\Omega_{M} / 0.25\right)^{0.47}$ parameter combination, taken from Vikhlinin et al. (2009b). In our work stronger constraints on $N_{\text {eff }}$ were obtained since we used complete likelihoods for galaxy cluster mass function data. Note also that, as compared to Keisler et al. (2011), systematic errors are not included in our upper limits on $N_{\text {eff }}$ (see discussion above).

Compatible constraints on $N_{\text {eff }}$ were obtained from the other data on galaxy cluster mass function. For example, in (Mantz et al., 2010b) the constraint $N_{\text {eff }}=3.4_{-0.5}^{+0.6}(68 \%$ c.1.) was obtained. Therefore, the constraints from the data on galaxy clusters are in better agreement with the standard value $N_{\text {eff }}=3.046$, as compared to $A C T$ and $S P T$ results. A significant constraint, which is also consistent with the standard value $N_{\text {eff }}=3.046$, was obtained from the comparison of observed abundance of light elements 

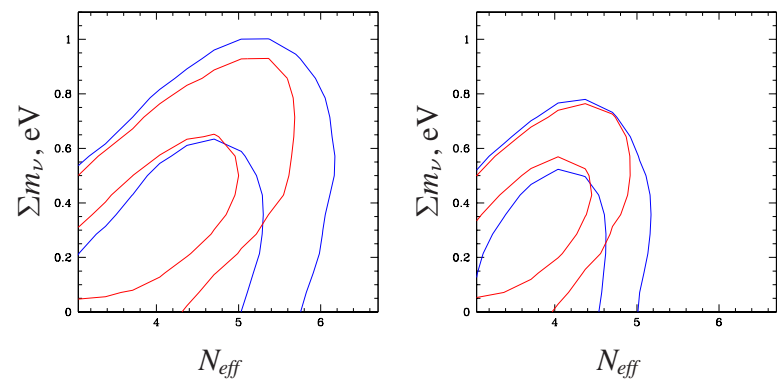

Fig. 9. Constraints on effective number of neutrino species and on total neutrino mass in $\Lambda C D M$ model with free $N_{\text {eff }}$ and $\Sigma m_{\nu}$. Larger contours correspond to $W M A P 7+B A O+H_{0}$ dataset (left) and $W M A P 7+B A O+H_{0}+S P T$ dataset (right), smaller contours - to the same data, with the data on galaxy cluster mass function added.

with the predictions of primordial nucleosynthesis theory (Mangano, Serpico, 2011).

We emphasize that our constraints on $N_{\text {eff }}$, and also all other constraints on this parameter, discussed above (except the constraint from observed light element abundance), were obtained in assumption of zero total neutrino mass. However, if both total neutrino mass and $N_{\text {eff }}$ are considered as free parameters, the constraints on both of them turn to be substantially weaker. This case is considered below.

\section{Effective number of massive neutrinos}

The constraints on total neutrino mass and effective number of neutrino species $N_{\text {eff }}$ in $\Lambda C D M$ model with arbitrary number of massive neutrinos are shown in Fig. 9 and 10 (see also Table 1). Note, that if arbitrary number of neutrino species are allowed in the model, the upper limits on their total mass turn to be substantially weaker. And vice-versa, the assumption on non-zero total neutrino mass weaken the upper limits on number of neutrino species. We obtain the following upper limits from $C L+W M A P 7+B A O+H_{0}+S P T$ dataset: $\Sigma m_{\nu}<0.72 \mathrm{eV}$ and $N_{\text {eff }}<4.62$.

Therefore, the most powerful set of cosmological data used in our work $\left(C L+W M A P 7+B A O+H_{0}+S P T\right)$ does not exclude the existence of one additional type of neutrino. Moreover, for these data the maximum likelihood is shifted to the model with total neutrino mass about $0.4 \mathrm{eV}$ and number of neutrino species $N_{\text {eff }} \approx 4$. The improvement of maximum likelihood for this model, as compared to the model with $\Sigma m_{\nu}=0$ and $N_{\text {eff }}=3.046$, is $\Delta \ln L=1.80$, which corresponds to $\Delta \chi^{2}=3.60$ for 2 degrees of freedom, approximately $1.4 \sigma$ significance. From Fig. 10 one can see that the likelihood is improved mainly due to the assumption on non-zero neutrino mass. When massive neutrinos are added to the model with free number of neutrino species, the likelihood improvement corresponds to $\Delta \chi^{2}=3.41$ for one degree of freedom, which corresponds to approximately $1.9 \sigma$ significance.
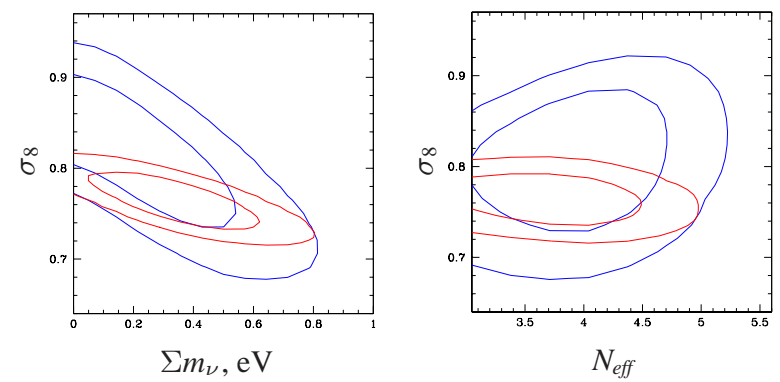

Fig. 10. Constraints on effective number of neutrino species and on total neutrino mass in $\Lambda C D M$ model with free $N_{\text {eff }}$ and $\Sigma m_{\nu}$. Larger contours correspond to WMAP7+BAO+H$+S P T$ dataset, smaller contours - to the same data, with the data on galaxy cluster mass function added.

Systematical errors of cluster mass measurements decrease the significance of introduction of these new parameters in the model. For example, if cluster masses are underestimated for $\delta M / M=0.09$, then the introduction of massive neutrinos into the $\Lambda C D M$ model with free $N_{\text {eff }}$ gives $\Delta \chi^{2}=2.83$ and significance about $1.7 \sigma$. One can see, that systematic uncertainties in cluster mass measurements are very significant in total neutrino mass constraints even with existing cluster data. Therefore, in order to improve similar measurements in future one will need to significantly reduce systematic uncertainties in cluster mass measurements.

\section{Different mass distributions of neutrino species}

All constraints on total neutrino mass, discussed above, were obtained in assumption that three known neutrino species have the same equal masses (and additional neutrinos are massless), i.e. the mass distribution of these three known neutrino species is degenerate. However, from the observations of neutrino oscillation it is known, that masses of different neutrino species are also different. Generally, if the mass distribution of neutrino species is non-degenerate, the constraints on total neutrino mass from cosmological data may change. This happens for several reasons (see, e.g., Slosar, 2006), in particular since most massive neutrino species become non-relativistic earlier and spectrum of linear density perturbations is suppressed at smaller scales. Also, if neutrino mass is larger then approximately $0.5 \mathrm{eV}$, they become non-relativistic before recombination and significantly change angular power spectrum of $\mathrm{CMB}$ anisotropy.

In case of $N_{\text {eff }}=3.046$, the assumption on nondegenerate neutrino mass distribution makes almost no change in the neutrino mass constraints from cosmological data. For example, if there is only one massive neutrino, the constraint on total neutrino mass from $C L+W M A P 7+B A O+H_{0}+S P T$ dataset weaken only very slightly, $\Sigma m_{\nu}<0.33 \mathrm{eV}$ (as compared to $\Sigma m_{\nu}<0.32 \mathrm{eV}$, in case of three degenerate neutrinos, see above). This is quite consistent with what is expected for similar cosmo- 

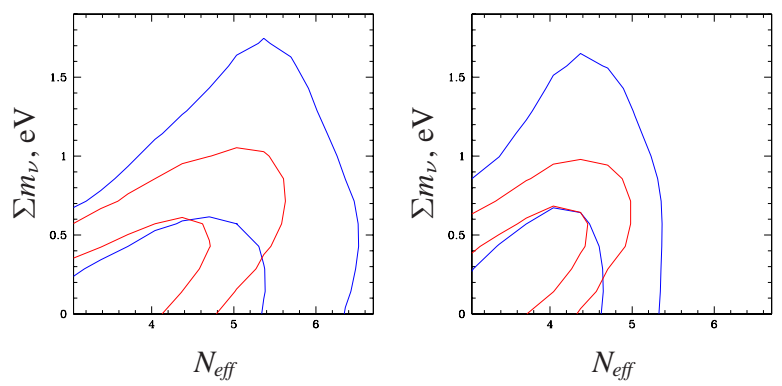

Fig. 11. The same constraints as in Fig. 9 but in assumption that there is only one type of neutrino with non-zero mass.

logical data (e.g., Takada et al., 2006; Slosar, 2006).

It turns out, that with increasing number of neutrino species, the assumption that only one neutrino is massive weaken the constraint on total neutrino mass more significantly. This is shown in Fig. 11 - the upper limit for $C L+W M A P 7+B A O+H_{0}+S P T$ dataset is $\Sigma m_{\nu}<0.88 \mathrm{eV}$ in this case. The upper limit on number of neutrino species changes only slightly and is $N_{\text {eff }}<4.68$. These upper limits can be compared to $\Sigma m_{\nu}<0.72 \mathrm{eV}$ and $N_{\text {eff }}<4.62$ in case of three degenerate neutrinos (see above).

\section{Light sterile neutrinos}

The constraints on $\Sigma m_{\nu}$ and $N_{\text {eff }}$ discussed above can have important application to study the possibility of existence of light sterile neutrinos with masses near $1 \mathrm{eV}$, which were suggested in order to explain the results of short baseline neutrino oscillations searches experiments LSDN (Aguilar et al., 2001) and MiniBooNE (Aguilar-Arevalo A. A. et al., 2010), and also recently discovered, so-called reactor neutrino anomaly (Mueller et al., 2011; Mention et al., 2011). In order to explain these results a possibility of the existence of one or two light sterile neutrino species (Maltoni, Schwetz, 2007; Karagiorgi et al., 2009; Melchiorri et al., 2009; Akhmedov, Schwetz, 2010; Kopp et al., 2011), which should be thermalized in early Universe (see, e.g., discussion and references in Hamann et al., 2010) was discussed. The presence of these additional neutrinos should also change the value of $N_{\text {eff }}$, measured from cosmological data.

The upper limit $N_{\text {eff }}<3.70$, which was obtained in our work in assumption on zero total neutrino mass (see above), does not agree with the existence of even one additional type of neutrino. However, in order to explain the results of short baseline neutrino oscillations experiments additional species of massive neutrinos are suggested (e.g., Mention et al., 2011; Kopp et al., 2011). In this case the constraints on $N_{\text {eff }}$ turn to be significantly weaker. As it was shown above, all considered cosmological data are consistent with the existence of one additional type of light sterile neutrino with mass about $0.4 \mathrm{eV}$.

It was discussed recently, that the results of short base- line neutrino oscillations searches are better explained if two additional light sterile neutrino species are suggested (Kopp et al., 2011; Giunti, Laveder, 2011a). However, recent improvements of the MiniBooNE experiment data, probably will allow to explain all the data of these experiments with only one additional light sterile neutrino (Giunti, Laveder, 2011b). Anyway, in order to explain the results of these experiment assuming only one additional type of neutrino, the masses near $1 \mathrm{eV}$ are probably required (Kopp et al., 2011; Giunti, Laveder, 2011b). This value disagree with upper limits on total neutrino mass, obtained above - even in most conservative case of one additional massive neutrino the upper limit on total neutrino mass is $\Sigma m_{\nu}<0.88 \mathrm{eV}$ at $95 \%$ confidence level. Therefore, we conclude that masses of light sterile neutrinos, which may explain current results of short baselines neutrino oscillation searches are not in good agreement with our constraints.

In order to reconcile neutrino masses near $1 \mathrm{eV}$ with the constraints on total neutrino mass from cosmological data it was suggested to consider the cosmological models with dark energy more general than cosmological constant (Kristiansen, Elgaroy, 2011; Hamann et al., 2011). It was found that with dark energy equation of state parameter $w<-1$ the limits on neutrino mass are relaxed considerably. This remains true also when the galaxy cluster mass function cosmological data taken in account. The upper limits on total neutrino mass and effective number of neutrino species in the model with free $w$, and free number of massive neutrinos are $\Sigma m_{\nu}<0.85 \mathrm{eV}$ and $N_{\text {eff }}<4.33$ in assumption that three neutrino species have equal non-zero mass, and $\Sigma m_{\nu}<1.00 \mathrm{eV}$ and $N_{\text {eff }}<4.39$ in case if there is only one type of massive neutrino in the model. Therefore, the limits on total neutrino mass, obtained with galaxy cluster mass function data taken in account, are still in poor agreement with suggested sterile neutrino mass near $1 \mathrm{eV}$, even in cosmological models with dark energy equation of state parameter $w<-1$.

Therefore, all available cosmological data are consistent with one additional light sterile neutrino. However, their total mass may be near $0.4 \mathrm{eV}$, while the values of total neutrino mass near $1 \mathrm{eV}$ are in poor agreement with existent cosmological data. We emphasize that the constraints obtained in our work apply only to light sterile neutrinos thermalized in early Universe.

\section{DARK ENERGY CONSTRAINTS}

In order to obtain most powerful constraints on dark energy equation of state, the measurements of accelerated expansion of Universe from the observations of distant supernovae type Ia were used in Komatsu et al. (2011). On the other hand, during the last years it become clear that the errors of cosmological measurements based on SN Ia data are dominated by systematic uncertainties (Hicken et al., 2009; Kessler et al., 2009). In recent works on SN Ia observa- 

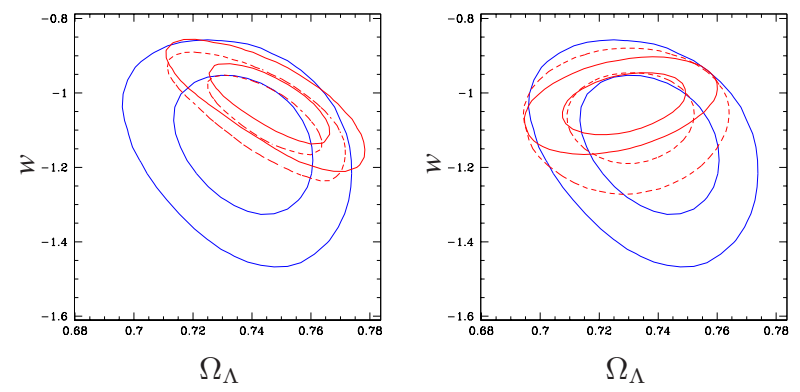

Fig. 12. The constraints on dark energy density and equation of state parameter in $W C D M$ model. Larger contours - for $W M A P 7+B A O+H_{0}$ dataset, smaller contours - for $W M A P 7+B A O+H_{0}+C L$ dataset (left panel) and for $W M A P 7+B A O+H_{0}+S N$ dataset (right panel). Dashed line in left panel show the contours for systematic $\delta M / M \approx 0.09$ shift in cluster mass measurements. In the right panel the contours for SN Ia data with systematic uncertainties taken in account are shown with dashed line.

tions, systematical errors are estimated to be very significant and to be larger than statistical errors in these data. These uncertainties are mostly consist of uncertainties of photometrical calibrations, color corrections on the extinction in host galaxies, selection effects in SN Ia surveys and others (e.g., Amanullah et al., 2010).

With these considerations in mind, it is interesting to study the possibilities to obtain powerful dark energy constraints independently on SN Ia measurements. We show below that these constraints may be obtained using galaxy cluster mass function measurements, and their uncertainties are not currently dominated by systematics. The updated dark energy constraints obtained from the combination of recent cosmological data are also given below.

\section{Flat Universe}

The constraints on the density and equation of state parameter $w$ of dark energy in a flat Universe with free $w$ (WCDM model) are shown in Fig. 12 and 13 (see also Table 1). With no systematic uncertainties taken in account, the existing data on galaxy cluster mass function give somewhat weaker constraints, as compared to the data on SN Ia. However, the error on $w$ parameter measured from from SN Ia data is dominated by systematic uncertainties (see right panel of Fig. 12). With systematical errors taken in account, the constraint on dark energy equation of state parameter from WMAP7+BAO+H$+S N$ dataset is $w=-1.068 \pm 0.077$. On the other hand, the $W M A P 7+B A O+H_{0}+C L$ dataset gives the following constraint: $w=-1.026 \pm 0.069$ (stat.) \pm 0.028 (sys.), i.e., the total error is \pm 0.074 in this case. Therefore, the constraints from galaxy cluster mass function data and from SN Ia observations are comparably powerful. Note that, in contrast to SN Ia data, the errors from cluster mass function measurements are not currently dominated by systematic uncertainties.

The constraints for all the data combined are shown in Fig. 13. One can see that galaxy cluster mass function

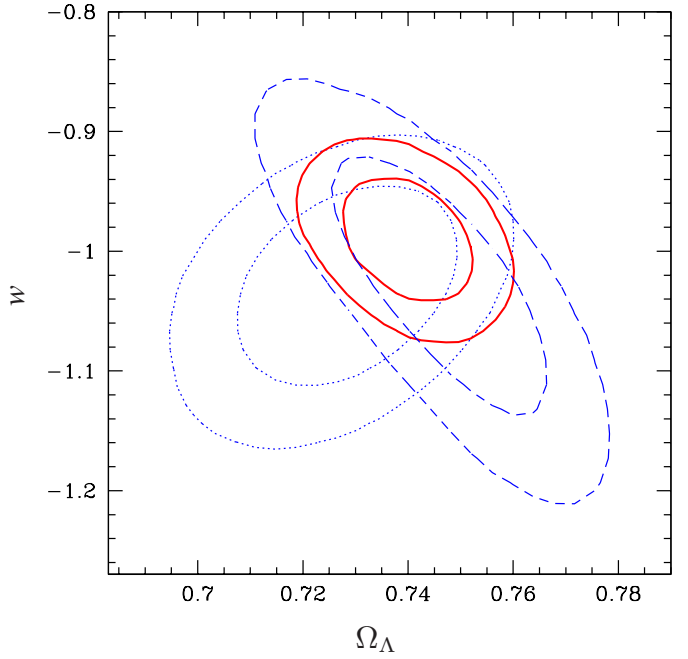

Fig. 13. The constraints on dark energy density and equation of state parameter in WCDM model. Larger contours - for WMAP7+BAO+ $\mathrm{H}_{0}+\mathrm{SN}$ dataset (dotted lines) and also for $W M A P 7+B A O+H_{0}+C L$ (dashed lines), smaller contours - for all the data $\left(W M A P 7+B A O+H_{0}+S N+C L\right)$ combined.
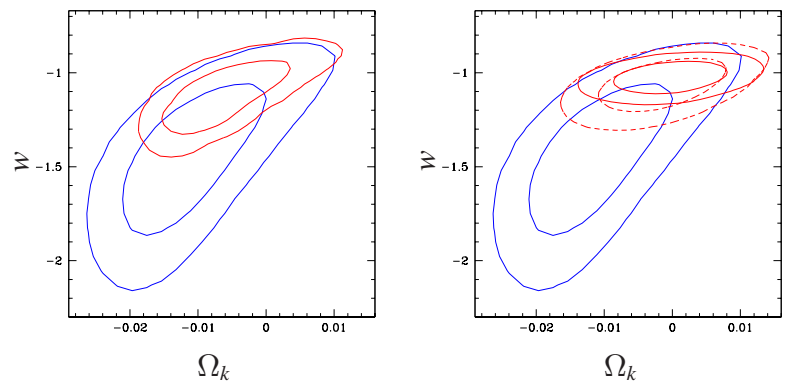

Fig. 14. The constraints on the curvature of Universe and dark energy equation of state parameter in $W C D M$ model with $\Omega_{k} \neq 0$. Larger contours - for $W M A P 7+B A O+H_{0}$ dataset, smaller contours - for the same data with the data on clusters (left panel) and SN Ia (right panel) added. The contours for SN Ia data with systematic uncertainties taken in account are shown with dashed lines in right panel of the Figure.

data significantly improve the dark energy equation of state parameter constraints. The reason is that these data are independent and have different degeneracies in parameter space. From these data we obtain the following measurement: $w=-0.990 \pm 0.034$ (stat.) \pm 0.041 (sys.), where SN Ia systematic uncertainties are also included in resulting systematic error. These constraints appears to be somewhat better than those obtained in Vikhlinin et al. (2009b).

\section{Curved Universe}

The constraint on the curvature of Universe and dark energy equation of state parameter in $W C D M$ model with $\Omega_{k} \neq 0$ are shown in Fig. 14 and 15 In model with nonzero space curvature the cluster data allow to obtain only less powerful constraint on $w$, as compared to supernovae data (cf. left and right panels in Fig. 14). However, taking in account systematic errors of supernovae data, the accu- 


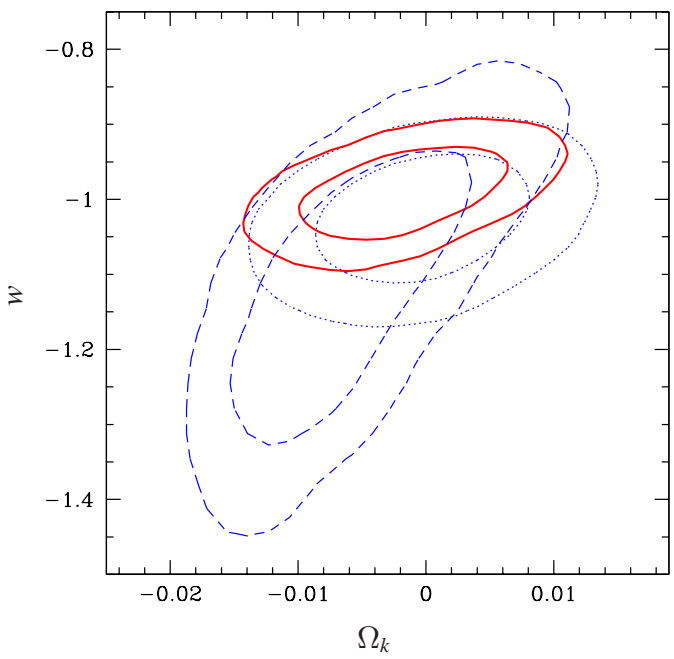

Fig. 15. The constraint on the curvature of Universe and dark energy equation of state parameter in WCDM model with $\Omega_{k} \neq 0$. Larger contours - for $W M A P 7+B A O+H_{0}+S N$ (dotted lines) and for $W M A P 7+B A O+H_{0}+C L$ dataset (dashed lines), smaller contours - for all the data $\left(W M A P 7+B A O+H_{0}+S N+C L\right)$ combined.

racy of $w$ measurement appears to be comparable. From Fig. 15 one can see that the data on galaxy cluster mass function combined with all other data, including the data on SN Ia, allow to significantly improve the measurement of the parameter of dark energy equation of state.

\section{Time-dependent equation of state}

The constraints on the parameters of time-dependent dark energy equation of state, defined as:

$$
w(a)=w_{0}+w_{a}(1-a),
$$

are shown in Fig. 16 (see also Table 1. WACDM model). In order to calculate CMB anisotropy power spectra we used Parametrized Post-Friedmann approach (Fang et al., 2008), which was done using the correspondent module for CosmoMc software. One can see, that galaxy cluster mass function data significantly improve the overall constraints for $w_{0}$ and $w_{a}$ parameters. Therefore, existent cluster mass function data give comparably powerful constraints for these parameters, as compared to supernovae data.

\section{SUMMARY}

In our work we present the results of detailed analysis of cosmological parameter constraints which were obtained from combination of galaxy cluster mass function measurements (Vikhlinin et al., 2009a,b) with the other cosmological data, obtained recently. We show, that this allow to significantly improve the constraints for many cosmological parameters.

All considered cosmological data combined together are consistent with the model of flat Universe with cosmolog-

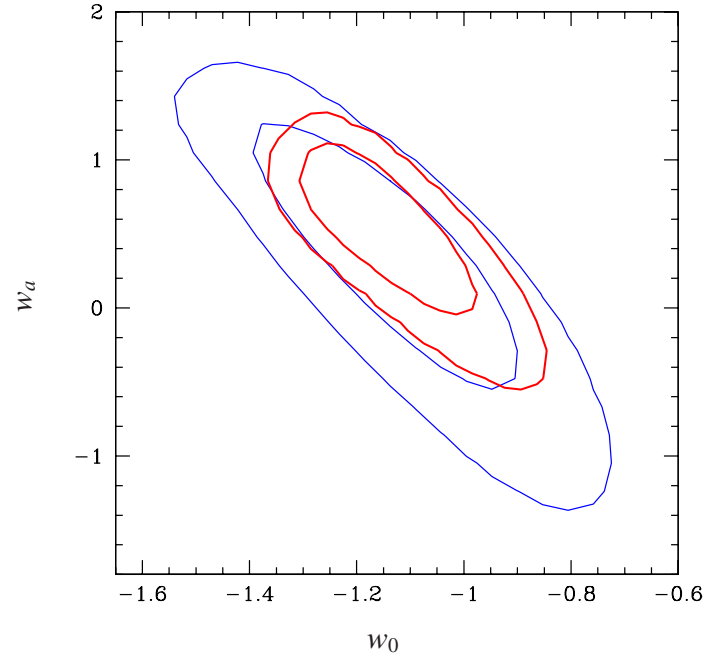

Fig. 16. The constraints on dark energy equation of state parameters in $W A C D M$ model. Larger contours - for $W M A P 7+B A O+H_{0}+S N$ dataset, smaller contours - for $W M A P 7+B A O+H_{0}+S N+C L$ dataset.

ical constant $(\Lambda C D M)$. In frames of this model all considered cosmological data, with galaxy cluster mass function data taken in account, give the most powerful constraints on $\sigma_{8}$ and $\Omega_{m} h^{2}$ parameters. The constraints on other parameters, such as $\Omega_{m}, H_{0}, \Omega_{b}$, are also improved. The data on galaxy cluster mass function allow to constrain these parameters with as high as $\approx 1 \%$ accuracy (see Table 1 ). At the same time, the systematical errors from the uncertainties of cluster mass measurements appear to be comparable to statistical ones.

The data on galaxy cluster mass function allow to obtain new important constraints on total neutrino mass $\Sigma m_{\nu}$ and effective number of neutrino species $N_{\text {eff }}$. When massive neutrinos or additional number of neutrino species are added to $\Lambda C D M$ model, the strongest constraints are obtained: $\Sigma m_{\nu}<0.32 \mathrm{eV}$ and $N_{\text {eff }}<3.74$. However, if both total neutrino mass and effective number of neutrino species are considered as free parameters in $\Lambda C D M$ model, i.e., the model with arbitrary number of massive neutrinos is considered, the constraints turn out to be significantly less strong: $\Sigma m_{\nu}<0.72 \mathrm{eV}$ and $N_{\text {eff }}<4.62$. Moreover, all considered cosmological data are somewhat better fitted by the model with non-zero neutrino mass $\Sigma m_{\nu} \approx 0.4 \mathrm{eV}$ and larger than standard value of the number of neutrino species, $N_{\text {eff }} \approx 4$.

These constraints can have an important application to study of the possibility of the existence of light sterile neutrinos with masses near $1 \mathrm{eV}$, which were suggested to explain the results of short baseline neutrino oscillations searches experiments $L S D N$ and MiniBooNE, and also recently discovered, so-called reactor neutrino anomaly. However, as compared to cosmological constraints obtained in our work, in order to explain the results of these experiments, too large number of additional neu- 
trino species (more than one) or too large neutrino masses $\Sigma m_{\nu} \approx 1 \mathrm{eV}$ are required.

In our work the updated constraints on dark energy equation of state parameters are also presented. The constraints obtained using galaxy cluster mass function data, with no use of SN Ia measurements, are considered as well. We show, that taking in account systematical uncertainties, dark energy parameters constraints from cluster mass function data are comparable in their power with those from SN Ia observations. Moreover, in contrast to SN Ia data, the errors of dark energy parameters measurements from current cluster mass function data are mainly statistical, and are not dominated by systematic uncertainties. Therefore, expansion of a sample of galaxy clusters, suitable for accurate measurements of galaxy cluster mass function, will allow to further improve dark energy constraints in future.

We are grateful to D. S. Gorbunov for useful discussion of the results of our work and for a number of important remarks and suggestions. In this work the results of calculations on MVS-100K supercomputer of Joint Supercomputer Center of the Russian Academy of Sciences (JSCC RAS) were used. The work is supported by Russian Foundation for Basic Research, grants 08-02-00974, 09-02-12384-ofi-m, 10-02-01442, 11-02-12271-ofi-m, the Program for Support of Leading Scientific Schools of the Russian Federation (Nsh-5603.2012.2), and the Programs of the Russian Academy of Sciences P-19 and OPhN-16.

\section{REFERENCES}

2. Abazajian K. N., Calabrese E., Cooray A., et al., Astroparticle Phys. 35, 177 (2011).

3. Aguilar A., Auerbach L. B., Burman R. L., et al., Phys. Rev. D 64, 112007 (2001).

4. Aguilar-Arevalo A. A., et al., The MiniBooNE Collaboration, Phys. Rev. Letters 105, 181801 (2010); arXiv: 1007.4730.

5. Amanullah R., Lidman C., Rubin D., Aldering G., Astier P., Barbary K., Astrophys. J. 716, 712 (2010).

6. Akhmedov E., Schwetz T., J. of High Energy Phys. 2010, 115 (2010).

7. Bolton J. S., Viel M., Kim T.-S., Haehnelt M. G., Carswell R. F., Mon. Not. R. Astron. Soc. 386, 1131 (2008).

8. Borgani S., Rosati P., Tozzi P., Stanford S. A., Eisenhardt P. R., Lidman C., et al., Astrophys. J. 561, 13 (2001).

9. Brandbyge J., Hannestad S., Haugbolle T., Wong Y. Y. Y., J. of Cosmology and Astroparticle Phys. 09, 014 (2010).

10. Brown M. L., Ade P., Bock J., Bowden M., Cahill G., Castro P. G., Astrophys. J. 705, 978 (2009).

11. Burenin R. A., Vikhlinin A., Hornstrup A., Ebeling H., Quintana H., Mescheryakov A., Astrophys. J. Suppl. Ser. 172, 561 (2007).

12. Chluba J., Sunyaev R. A., Astron. Astrophys. 446, 39 (2006).

13. Chluba J., Sunyaev R. A., Astron. Astrophys. 475, 109 (2007).
14. Chluba J., Sunyaev R. A., Astron. Astrophys. 503, 345 (2009).

15. Chluba J., Sunyaev R. A., Astron. Astrophys. 512, A53 (2010).

16. Dolgov A. D., Hansen S. H., Semikoz D. V., Nuclear Phys. B 543, 269 (1999).

17. Doroshkevich A. G., Khlopov M. Iu., Sunyaev R. A., Szalay A. S., Zeldovich Ia. B., Texas Symposium on Relativistic Astrophysics, 10th, Baltimore, MD, Dec. 1519, 1980.) New York Academy of Sciences, Annals, 375, 32 (1981).

18. Dunkley J., Hlozek R., Sievers J., Acquaviva V., Ade P. A. R., Aguirre P., et al., Astrophys. J. Suppl. Ser., in press, arXiv: 1009.0866 .

19. Dubrovich V. K., Grachev S. I., Pis'ma v Astron. Zhurn. 31, 403 (2005) [Astronomy Letters, 31, 359].

20. Fang W., Hu W., Lewis A., Phys. Rev. D 78, 087303 (2008).

21. Freedman W. L., Madore B. F., Gibson B. K., Ferrarese L., Kelson D. D., Sakai S., et al., Astrophys. J. 553, 47 (2001).

22. Gnedin N. Y., Gnedin O. Y., Astrophys. J. 509, 11 (1998).

23. Gorbunov D. S., Rubakov V. A., "Introduction in the Theory of Early Universe. Cosmologicl perturbations. Theory of Inflation.", Moscow: KRASAND, 2010 (in Russian).

24. Giunti C., Laveder M., Phys. Rev. D 84, 7 (073008).

25. Giunti C., Laveder M., arXiv:1109.4033.

26. Hamann J., Hannestad S., Raffelt G., Tamborra I., Wong Y. Y. Y., Phys. Rev. D 105, 181301 (2010).

27. Hamann J., Hannestad S., Raffelt G., Wong Y. Y. Y., J. of Cosmology and Astroparticle Phys. 09, 034 (2011).

28. Haiman Z., Mohr J. J., Holder G. P., Astrophys. J. 553, 545 (2001).

29. Henry J. Patrick, Astrophys. J. (Letters) 534, 565 (2000).

30. Henry J. Patrick, Astrophys. J. (Letters) 609, 603 (2004).

31. Hicken M., Wood-Vasey W. M., Blondin S., Challis P., Jha S., Kelly P. L., Rest A., Kirshner R. P., Astrophys. J. 700, 1097 (2009).

32. Hoekstra H., Mon. Not. R. Astron. Soc. 379, 317 (2007).

33. Hu W., Eisenstein D. J., Tegmark M., Phys. Rev. Letters 80, 5255 (1998).

34. Hu W., Dodelson, S., Ann. Rev. of Astron. and Astrophys. 40, 171 (2002).

35. Hu W., Jain B., Phys. Rev. D 70, 043009 (2004).

36. Karagiorgi G., Djurcic Z., Conrad J. M., Shaevitz M. H., Sorel M., Phys. Rev. D 80, 073001 (2009).

37. Keisler R., Reichardt C. L., Aird K. A., Benson B. A., Bleem L. E., Carlstrom J. E., et al., Astrophys. J., in press; arXiv: 1105.3182.

38. Kessler R., Becker A. C., Cinabro D., Vanderplas J., Frieman J. A., Marriner J., et al., Astrophys. J. 700, 1097 (2009).

39. Kholupenko E. E., Ivanchik A. V., Varshalovich D. A., Mon. Not. R. Astron. Soc. 378, L39 (2007).

40. Kholupenko E. E., Ivanchik A. V., Varshalovich D. A., Pis'ma v Astron. Zhurn. 34, 803 (2008) [Astronomy Letters, 34, 725]. 
41. Komatsu E., Smith K. M., Dunkley J., Bennett C. L., Gold B., Hinshaw G., et al., Astrophys. J. Suppl. Ser. 192, 18 (2011).

42. Kopp J., Maltoni M., Schwetz T., Phys. Rev. Letters 107, 091801 (2011); arXiv:1103.4570.

43. Kristiansen J. R., Elgaroy O., Astron. Astrophys. 532, A67 (2011).

44. Larson D., Dunkley J., Hinshaw G., Komatsu E., Nolta M. R., Bennett C. L., et al., Astrophys. J. Suppl. Ser. 192, 16 (2011).

45. Lewis A., Challinor A., Lasenby A., Astrophys. J. 538, 473 (2000).

46. Lewis A., Bridle S., Phys. Rev. D 66, 103511 (2002).

47. Majumdar S., Mohr J. J., Astrophys. J. 613, 41 (2004),

48. Malinovsky A. M., Voevodkin A. A., Lukash V. N., Mikheeva E. V., Vikhlinin A. A., Pis'ma v Astron. Zhurn. 34, 490 (2008); [Astronomy Letters, 34, 445].

49. Maltoni M., Schwetz T., Phys. Rev. D 76, 093005 (2007).

50. Mangano G., Miele G., Pastor S., Peloso M., Physics Lett. B 534, 8 (2002).

51. Mangano G., Serpico P. D., Physics Lett. B 701, 296 (2011); arXiv:1103.1261.

52. Mantz A., Allen S. W., Ebeling H., Rapetti D., Mon. Not. R. Astron. Soc. 387, 1179 (2008).

53. Mantz A., Allen S. W., Ebeling H., Rapetti D., Mon. Not. R. Astron. Soc. 406, 1759 (2010Đř).

54. Mantz A., Allen S. W., Rapetti D., Mon. Not. R. Astron. Soc. 406, 1805 (2010Đś).

55. Marulli F., Carbone C., Viel M., Moscardini L., Cimatti A., Mon. Not. R. Astron. Soc., in press (2011); arXiv:1103.0278.

56. Melchiorri A., Mena O., Palomares-Ruiz S., et al., J. of Cosmology and Astroparticle Phys. 01, 036 (2009).

57. Mention G., Fechner M., Lasserre Th., et al., Phys. Rev. D 83, 073006 (2011).

58. Mueller Th. A., Lhuillier D., Fallot M., et al., Phys. Rev. C 83, 054615 (2011).

59. Percival W. J., Reid B. A., Eisenstein D. J., Bahcall N. A., Budavari T., Frieman J. A., et al., Mon. Not. R. Astron. Soc. 401, 2148 (2010).

60. Reichardt C. L., Ade P. A. R., Bock J. J., Bond J. R., Brevik J. A., Contaldi C. R., et al., Astrophys. J. 694, 1200 (2009).

61. Reid B. A., Verde L., Jimenez R., Mena O., J. of Cosmology and Astroparticle Phys. 01, 003 (2010).

62. Reiprich, T. H., Böhringer H., Astrophys. J. 567, 716 (2002).

63. Riess A. G., Macri L., Casertano S., Sosey M., Lampeit H., Ferguson H. C., Filippenko A. V., Jha S. W., Li W., Chornock R., Sarkar D., Astrophys. J. 699, 539 (2009).

64. Riess A. G., Macri L., Casertano S., Lampeit H., Ferguson H. C., Filippenko A. V., Jha S. W., Li W., Chornock R., Silverman J., Astrophys. J. 732, 129 (2011).

65. Rubino-Martin J. A., Chluba J., Fendt W. A., Wandelt B. D., Mon. Not. R. Astron. Soc. 403, 439 (2010).

66. Schuecker P., Böhringer H., Collins C. A., Guzzo L., Astron. Astrophys. 398, 867 (2003).
67. Seljak U., Slosar A., McDonald P., J. of Cosmology and Astroparticle Phys. 10, 014 (2006).

68. Seager S.,Sasselov D. D., Scott D., Astrophys. J. 523, L1 (1999).

69. Slosar A., Phys. Rev. D 73, 123501 (2006).

70. Starobinsky A. A., ZhETF Letters 68, 721 (1998).

71. Sunyaev R. A., Chluba J., Astronomische Nachrichten, 330, 657 (2009).

72. Takada M., Komatsu E., Futamase T., Phys. Rev. D 73, 083520 (2006).

73. Tinker J., Kravtsov A. V., Klypin A., Abazajian K., Warren M., Yepes G., GottlÃúber S., Holz D. E., Astrophys. J. 688, 709 (2008).

74. Thomas S. A, Abdalla F. B., Lahav O., Phys. Rev. Letters 105, 031301 (2010).

75. Vanderlinde K., Crawford T. M., de Haan T., Dudley J. P., Shaw L., Ade P. A. R., et al., Astrophys. J. 722, 1180 (2010).

76. Vikhlinin A., Voevodkin A., Mullis C. R., van Speybroeck L., Quintana H., McNamara B. R., Gioia I., Hornstrup A., Henry J. P., Forman W. R., Jones C., Astrophys. J. 590, 15 (2003).

77. Vikhlinin A., Burenin R. A., Ebeling H., Forman W. R., Hornstrup A., Jones C., Kravtsov A. V., Murray S. S., Nagai D., Quintana H., Voevodkin A., Astrophys. J. 692, 1033 (2009Đř).

78. Vikhlinin A., Kravtsov A. V., Burenin R. A., Ebeling H., Forman W. R., Hornstrup A., Jones C., Murray S. S., Nagai D., Quintana H., Voevodkin A., Astrophys. J. 692, 1060 (2009Đś).

79. Vikhlinin A., Publications of the National Academy of Science 107, 16 (2010).

80. Voevodkin A., Vikhlinin A., Astrophys. J. 601, 610 (2004).

81. Wang S., Khoury J., Haiman Z., May M., Phys. Rev. D 70, 123008 (2004).

82. Weller J., Battye R. A., Kneissl R., Phys. Rev. Letters 88, 231301 (2002).

83. Zhang Y.-Y., Finoguenov A., Böhringer H. et al., Astron. Astrophys. 482, 451 (2008). 